\title{
Ideal gas sources for the Lemâitre-Tolman-Bondi metrics
}

\author{
Roberto A. Sussman \\ Instituto de Ciencias Nucleares, UNAM, Apartado Postal 70-543, México D.F. \\ 04510, MÉXICO
}

\begin{abstract}
New exact solutions emerge by replacing the dust source of the Lemâitre-Tolman-Bondi metrics with a viscous fluid satisfying the monatomic gas equation of state. The solutions have a consistent thermodynamical interpretation. The most general transport equation of Extended Irreversible Thermodynamics is satisfied, with phenomenological coefficients bearing a close resemblance to those characterizing a non relativistic Maxwell-Bolzmann gas.
\end{abstract}

\section{Introduction.}

Dust is the prefered matter source in general relativistic models if rest mass is the dominant form of matter-energy ${ }^{[1,2]}$. Exact solutions with a dust source might provide an appropriate description of the dynamics of astrophysical systems, but lack any information on their thermal properties and evolution. A more complete description can be achieved by the replacement of the dust source with a nonrelativistic monatomic ideal gas. Since natural processes are irreversible and ideal gases (in general) are characterized by non-zero transport coefficients, a hydrodynamic model of this type of source should be based on the momentum energy tensor of an imperfect fluid. The purpose of this paper is to present a new class of exact solutions in which such matter tensor, subjected to the ideal gas equation of state, is the source of the Lemâitre-Tolman-Bondi (LTB) metrics ${ }^{[1,2]}$. However, Einstein field equations applied to these metrics (in comoving coordinates) necessarily restrict the fluid tensor to zero heat conduction and a non-accelerating 4-velocity, so that shear viscosity, associated with an anisotropic pressure tensor, becomes the only dissipative flux and the resulting shear viscosity tensor must be of a very special kind: its divergence must exactly ballance a non-zero gradient of the equilibrium pressure. Since, in general, classical ideal gases have non-zero heat conduction and pressure gradients produce non-zero 4-acceleration, the lack of these features obviously limits the physical applicability of the models, but is justified by the mathematical simplification of the field equations. Fortunately, though, the above mentioned limitations are compensated by the obtension of exact solutions that are thermodynamicaly consistent: the models derived in this paper are wholy compatible with the requirements (when shear viscosity is the only dissipative flux) of theories dealing with irreversible phenomena, such as Extended Irreversible Thermodynamics ${ }^{[3-10]}$ and Kinetic Theory ${ }^{[3,11]}$. Bearing in mind their shortcomings, these fluid models of a classical ideal gas, evolving along adiabatic (zero heat conduction) but irreversible (non-zero viscosity) thermodynamical processes ${ }^{[11,12]}$ can be considered a reasonable initial improvement on dust sources in the description of astrophysical systems in classical conditions. 


\section{Monatomic ideal gas sources.}

A non-relativistic, non-degenerated, monatomic ideal gas is characterized by the equation of state ${ }^{[7,9]}$

$$
\rho=m c^{2} n+\frac{3}{2} n k T, \quad p=n k T
$$

where $\rho, n, p, T$ are matter-energy and particle number densities, equilibrium pressure and temperature, $m, k$ are the particles' mass and Boltzmann's constant. As mentioned in the previous section, the hydrodynamical description of this ideal gas will be provided by solving Einstein's field equations for the momentum-energy tensor of a fluid with dissipative fluxes

$$
\begin{aligned}
& T^{a b}=\rho u^{a} u^{b}+p h^{a b}+\Pi^{a b}+2 c^{-1} q^{(a} u^{b)}, \\
& h^{a b}=c^{-2} u^{a} u^{b}+g^{a b}, \quad u_{a} \Pi^{a b}=0, \quad \Pi_{a}^{a}=0, \quad u_{a} q^{a}=0,
\end{aligned}
$$

where the equilibrium variables $\rho, p$ satisfy (1), $u^{a}$ is the 4-velocity of the particle frame ${ }^{[5]}, \Pi^{a b}$ is the shear viscous pressure and $q^{a}$ is the heat conduction vector (bulk viscosity is negligible for a classical ideal gas ${ }^{[3,9]}$. This matter tensor must satisfy energy-momentum ballance, as well as matter conservation and entropy production laws

$$
\begin{aligned}
& T_{; b}^{a b}=0 \\
& \left(n u^{a}\right)_{; a}=0 \\
& \left(n s u^{a}\right)_{; a} \geq 0
\end{aligned}
$$

where $s$ is the entropy per particle, which together with $q_{a}$ and $\Pi_{b}^{a}$ must comply with suitable transport and constitutive equations of Irreversible Thermodynamics.

\section{A class of exact solutions.}

Consider the Lemâitre-Tolman-Bondi (LTB) metric ansatz associated with spherically symmetric dust solutions ${ }^{[1,2]}$

$$
d s^{2}=-c^{2} d t^{2}+\frac{Y^{\prime 2}}{1-k_{0} f^{2}} d r^{2}+Y^{2}\left(d \theta^{2}+\sin ^{2} \theta d \phi^{2}\right)
$$

where $k_{0}=0, \pm 1, Y=Y(t, r), f=f(r)$ and $Y^{\prime}=Y_{, r}$. Following the strategy outlined previously, we shall consider the fluid tensor (2) (instead of dust) as the matter source of (4). If the spacial coordinates in (4) are comoving, we have $u^{a}=c \delta_{t}^{a}$, and so, the 4-velocity is a geodesic field $\left(u_{a ; b} u^{b}=0\right)$ characterized by two kinematical invariants: the expansion scalar $\Theta=u_{; a}^{a}$ and the shear tensor $\sigma_{a b}=u_{(a ; b)}-(1 / 3) \Theta h_{a b}$, given explicitly by

$$
\Theta=\frac{2 \dot{Y}}{Y}+\frac{\dot{Y}^{\prime}}{Y^{\prime}}
$$


$\sigma_{b}^{a}=\operatorname{diag}[0,-2 \sigma, \sigma, \sigma], \quad \sigma_{a b} \sigma^{a b}=6 \sigma^{2}, \quad \sigma=\frac{1}{3}\left(\frac{\dot{Y}}{Y}-\frac{\dot{Y}^{\prime}}{Y^{\prime}}\right)$

where $\dot{Y}=u^{a} Y_{, a}=c Y_{, t}$. The field equations for (2) and (4) imply vanishing heat coinduction: $q_{a}=q(t, r) \delta_{a}^{r}=0$, while the shear viscosity tensor in (2) (a traceless symmetric tensor) takes the form

$$
\Pi_{b}^{a}=\operatorname{diag}[0,-2 P, P, P], \quad \Pi_{a b} \Pi^{a b}=6 P^{2}
$$

where $P=P(t, r)$ is an arbitrary function to be determind by the field and transport equations. If $q_{a}=\dot{u}_{a}=0$ the ballance equation (3a) yields

$$
\begin{aligned}
& \dot{\rho}+(\rho+p) \Theta+\sigma_{a b} \Pi^{a b}=0 \quad \Rightarrow \quad \dot{p}+\frac{5}{3} \Theta p+4 \sigma P=0 \\
& h_{a}^{b}\left(p_{, b}+\Pi_{b c ; d} h^{c d}\right)=0 \quad \Rightarrow \quad-p^{\prime}+2 P^{\prime}+6 P \frac{Y^{\prime}}{Y}=0
\end{aligned}
$$

where $\dot{\rho}=u^{a} \rho_{, a}, \dot{p}=u^{a} p_{, a}$, the quantities $\sigma, P$ are defined in (5) and (6), and the relation $\dot{n}+n \Theta=0$ from (3b), as well as (1), were used to derive the expressions in the rhs of the implication sign.

Einstein's field equations for (2), (4) and (6), together with the integration of the matter conservation equation $(3 \mathrm{~b})$, yield

$$
\begin{aligned}
& \dot{Y}^{2}=\frac{8 \pi G}{3 c^{2} Y}\left[M+W\left(\frac{Y_{0}}{Y}\right)^{2}\right]-k_{0} c^{2} f^{2} \\
& \text { where: } \quad M \equiv m c^{2} \int n_{0} Y_{0}^{2} Y_{0}^{\prime} d r, \quad W \equiv \frac{3}{2} \int n_{0} k T_{0} Y_{0}^{2} Y_{0}^{\prime} d r
\end{aligned}
$$

and the subindex " 0 " below any function (as in $n_{0}, T_{0}, Y_{0}$ ) will denote henceforth evaluation of the function along a suitable intial hypersurface $t=t_{0}$. With the help of (8) and (9), the thermodynamical variables and $\sigma$ are given by

$$
\begin{gathered}
n=n_{0}\left(\frac{Y_{0}}{Y}\right)^{3} \frac{Y_{0}^{\prime} / Y_{0}}{Y^{\prime} / Y} \\
T=T_{0}\left(\frac{Y_{0}}{Y}\right)^{2} \Psi \\
p=n_{0} k T_{0}\left(\frac{Y_{0}}{Y}\right)^{5} \frac{Y_{0}^{\prime} / Y_{0}}{Y^{\prime} / Y} \Psi \\
P=\frac{1}{2} n_{0} k T_{0}\left(\frac{Y_{0}}{Y}\right)^{5} \frac{Y_{0}^{\prime} / Y_{0}}{Y^{\prime} / Y} \Omega \\
\frac{\dot{Y}}{Y} \frac{Y^{\prime}}{Y} \sigma=-\frac{4 \pi G}{9} m n\left[\Gamma+\frac{3}{2} \frac{k T_{0}}{m c^{2}}\left(\frac{Y_{0}}{Y}\right)^{2} \Omega\right] \frac{Y^{\prime}}{Y}+\frac{k_{0} c^{2} f^{2}}{3 Y_{0}^{2}}\left(\frac{Y_{0}}{Y}\right)^{2}\left(\frac{f^{\prime}}{f}-\frac{Y^{\prime}}{Y}\right)
\end{gathered}
$$

where:

$$
\Psi \equiv 1+\frac{2 W}{W^{\prime}}\left(\frac{Y_{0}^{\prime}}{Y_{0}}-\frac{Y^{\prime}}{Y}\right), \quad \Omega \equiv 1+\frac{W}{W^{\prime}}\left(\frac{2 Y_{0}^{\prime}}{Y_{0}}-\frac{5 Y^{\prime}}{Y}\right), \quad \Gamma \equiv 1-\frac{3 M}{M^{\prime}} \frac{Y^{\prime}}{Y}
$$


The solutions characterized by (1)-(15) become fully determined once (8) is integrated for specific initial conditions prescribed by selecting $n_{0}, T_{0}, Y_{0}, f$ (one of these functions can always be eliminated by an arbitrary rescaling of $r$ ). Ballance equations are identicaly satisfied, this follows by substitution of (5), (11), (12) into (7). Two important limiting cases emerge: (a) Lemâitre-Tolman-Bondi dust solutions are the zero temperature limit $\left(T_{0}=0 \Rightarrow T=p=P=0\right)$, and (b) The shear-free particular case ( $\sigma=0$, for all fluid worldlines) is the FLRW metric characterized by $f^{\prime} / f=Y^{\prime} / Y$, $Y=R(t) f, Y_{0}=R_{0} f, n_{0}^{\prime}=T_{0}^{\prime}=0$, whose source is a perfect fluid $(P=0)$ satisfying the equation of state $(1)^{[13]}$.

\section{Thermodynamical consistency.}

After integrating the field equations (pending the integration of (8)), it is necessary to examine the thermodynamical consistency of the viscous fluid source. For such a fluid, Extended Ireversible Thermodynamics associates a generalized entropy current ${ }^{[3-10]}$ satisfying (3c) and relating the deviation from equilibrium due to viscosity

$$
\left(n s u^{a}\right)_{; a} \geq 0 \Rightarrow \dot{s} \geq 0, \quad s=s^{(e)}-\alpha \pi_{a b} \pi^{a b}
$$

where $s^{(e)}$ follows from the integration of the equilibrium Gibbs equation $(T \mathrm{~d} s=$ $\mathrm{d}(\rho / n)+p \mathrm{~d}(1 / n))$ and $\alpha$ is a phenomenological coefficient. The evolution of the viscous pressure is in turn described by the transport equation ${ }^{[8-10]}$

$$
\tau \dot{\Pi}_{c d} h_{a}^{c} h_{b}^{d}+\Pi_{a b}\left[1+\frac{1}{2} T \eta\left(\frac{\tau}{T \eta} u^{c}\right)_{; c}\right]+2 \eta \sigma_{a b}=0
$$

where $\dot{\Pi}_{a b} \equiv \Pi_{a b ; c} u^{c}$, and the phenomenological quantities $\tau, \eta$ are the relaxation time and the coefficient of shear viscosity. Equation (17) is the most general transport equation available when shear viscosity is the only dissipative effect. In order to deal with a mathematicaly simpler problem, less general equations are often suggested. For example, Eckart's equation $(\tau=0)$ or the "truncated form" without the term involving $u^{c}$ and $T$ in (17). However, the former leads to acausal propagation equations and unstable solutions ${ }^{[4,9]}$, while numerical studies in FLRW spacetimes and other theoretical arguments ${ }^{[8-10]}$ point to unphysical effects in truncated forms of viscosity transport equations.

In order to solve equations (16) and (17) for (1)-(15), the coefficients $\alpha, \eta, \tau$ must be derived or specified. As a convenient reference providing a guideline on how to infere physicaly reasonable forms of these quantities, consider a classical monatomic ideal gas characterized by a Maxwell-Boltzmann distribution near equilibrium ${ }^{[3]}$. Grad's fourteen moments method provides the following forms for $\alpha, \eta, \tau$ for this ideal gas

$$
\tau_{M B}=\frac{1}{\gamma_{0} n} \quad(a), \quad \eta_{M B}=p \tau_{M B} \quad(b), \quad \alpha_{M B}=\frac{\tau_{M B}}{4 \eta_{M B} n T}=\frac{k}{4 p^{2}} \quad(c),
$$

where $\gamma_{0}$ is a collision integral (eq (3.41) of [3]). A reasonable test of the physical viability of the solutions follows from verifying if the forms of $n, T, p, \Pi_{a b}$ and $\sigma_{a b}$ in (5), (6), (11)-(15) are compatible with equations (16) and (17) in which the phenomenological coefficients are given by (18). This is examined below. 
Substitution of (11)-(13) into (16), with $\alpha$ given by (18c) and $s^{(e)}$ obtained from the integration of the equilibrium Gibbs equation for (1), yields

$s=s_{0}+k \ln \left[\left(\frac{T}{T_{0}}\right)^{3 / 2} \frac{n_{0}}{n}\right]-\frac{3}{2} k\left(\frac{P}{p}\right)^{2}=s_{0}+k \ln \left[\frac{Y^{\prime} / Y}{Y_{0}^{\prime} / Y_{0}} \Psi^{3 / 2}\right]-\frac{3}{8} k\left(\frac{\Omega}{\Psi}\right)^{2}$

where $s_{0}(r)$ is an arbitrary initial value of $s$. Regarding (17), if we assume: $\eta=p \tau$, with $p$ given by (12), then this transport equation is satisfied for $\tau$ given by:

$\tau=-\frac{\Omega \Psi}{4 \sigma}\left[\left[\frac{2 W}{W^{\prime}} \frac{Y^{\prime}}{Y}-\frac{7}{16}\left(1+\frac{2 W}{W^{\prime}} \frac{Y_{0}^{\prime}}{Y_{0}}\right)\right]^{2}+\frac{207}{256}\left(1+\frac{2 W}{W^{\prime}} \frac{Y_{0}^{\prime}}{Y_{0}}\right)^{2}\right]^{-1}$

While the form of $\eta$ can be justified as being formaly identical to the MaxwellBoltzmann form (18b) (a remarkable fact), the form of $\tau$ in (20) is acceptable as long as this expression behaves as a relaxation parameter for the system, namely: it should be a positive function and its relation with $\dot{s}$ (computed from (19)) must be such that it garantees $\dot{s} \geq 0$ along $u^{a}$ for all fluid worldlines. Idealy, of course, $\tau$ should be related to the mean collision time of the gas and should also, somehow, relate to or approach the Maxwell-Boltzmann form (18a). Evaluating $\dot{s}$ from (19) and comparing with (20), we find the following simple and elegant relation between $\dot{s}$ and $\tau$

$$
\dot{s}=\frac{3}{4} k\left(\frac{\Omega}{\Psi}\right)^{2} \frac{1}{\tau}=3 k\left(\frac{P}{p}\right)^{2} \frac{1}{\tau}
$$

which implies: $\dot{s}>0 \Leftrightarrow \tau>0$. This relation is in fact a direct consequence of the general expression: $\dot{s}=\Pi_{a b} \Pi^{a b} /(2 \eta n T)$ (see [4], eqn. (18)), together with equations (3b), (3c) and (18b). Therefore, from equations (11) and (19)-(21), the following are necessary and sufficient conditions for $T>0, \tau>0, \dot{s}>0$

$$
\begin{aligned}
& \Psi>0 \\
& \sigma \Omega<0
\end{aligned}
$$

while the conditions insuring that $\dot{s}$ decreases for increasing $\tau(\dot{\tau}>0 \Leftrightarrow \ddot{s}<0)$ follow from (20)-(21)

$$
\dot{\tau}>0, \quad \frac{\ddot{s}}{\dot{s}}=\frac{9 \sigma}{\Psi \Omega} \frac{W}{W^{\prime}} \frac{Y^{\prime}}{Y}\left(1+\frac{2 W}{W^{\prime}} \frac{Y_{0}^{\prime}}{Y_{0}}\right)-\frac{\dot{\tau}}{2 \tau}<0
$$

The set (22) provides the necessary and sufficient conditions for a theoreticaly consistent thermodynamical description of the solutions, within the framework of equations (16)-(21). However, in order to work out these conditions in a more explicit and direct manner (especialy (22c)), we need to know the explicit form of $Y^{\prime} / Y$ from integrating (8). 
5. The case $k_{0}=0$.

Consider the integration of (8). For the simplest case, $k_{0}=0$ (the cases $k_{0}= \pm 1$ are examined in [14]), this yields

$\frac{3}{2} \sqrt{\frac{8 \pi G M}{3 c^{2} Y_{0}^{3}}}\left(t-t_{0}\right)=\sqrt{y\left(y^{2}+\delta\right)}-\sqrt{1+\delta}+\frac{\delta^{3 / 4}}{2}\left(\mathrm{~F}-\mathrm{F}_{0}\right)$

$\frac{Y^{\prime}}{Y}=$

$\frac{M^{\prime}}{3 M}\left\{1-\tilde{\Gamma} \frac{\sqrt{y^{2}+\delta}}{y^{5 / 2} \sqrt{1+\delta}}+\frac{9}{4} \frac{k T_{0}}{m c^{2}} \tilde{\Omega}\left[\frac{\sqrt{y^{2}+\delta}}{y^{5 / 2}}\left(\frac{2 \delta}{\sqrt{1+\delta}}-\delta^{3 / 4}\left(\mathrm{~F}-\mathrm{F}_{0}\right)\right)-\frac{2 \delta}{y^{2}}\right]\right\}(23$

where: $\quad \tilde{\Gamma} \equiv 1-\frac{3 M}{M^{\prime}} \frac{Y_{0}^{\prime}}{Y_{0}}, \quad \tilde{\Omega} \equiv 1+\frac{W}{W^{\prime}}\left(\frac{2 Y_{0}^{\prime}}{Y_{0}}-\frac{5}{3} \frac{M^{\prime}}{M}\right)$

$\delta \equiv W / M, y \equiv Y / Y_{0}$ and $\mathrm{F}$ is the elliptic integral of the first kind with modulus $1 / \sqrt{2}$ and argument $\varphi$ given by: $\cos \varphi=(y-\sqrt{\delta}) /(y+\sqrt{\delta})$. With the help of $(23 \mathrm{~b})$ and the definitions of $\Psi, \Omega, \sigma$, it is possible to provide specific examples showing that conditions (22) are satisfied for physicaly reasonable initial value functions $n_{0}, T_{0}, Y_{0}$. Consider the following trial functions, analogous to empirical models of mass distribution in spherical gallaxies and clusters ${ }^{[15,16]}$

$n_{0}=\bar{n}_{0}\left[1+\frac{a}{1+\left(Y_{0} / R_{0}\right)^{2}}\right], \quad T_{0}=\bar{T}_{0}\left[1+\frac{b}{1+\left(Y_{0} / R_{0}\right)^{2}}\right], \quad Y_{0}=R_{0} r$

where $\bar{n}_{0}, \bar{T}_{0}, a, b$ are positive empirical constants, while $R_{0}$ is a characteristic length. Let us assume that (24) provides the initial particle number density and temperature profile at the time when matter and radiation decouple, $t_{0}=t_{d e c}$., of a spherical inhomogeneity of gallactic mass $\left(10^{44} \mathrm{gm}\right)$ in an expanding FLRW background characterized by an intial profile: $\bar{n}_{0} \approx 10^{5} \mathrm{~cm}^{-3}$ and $\bar{T}_{0} \approx 3 \times 10^{3} \mathrm{~K}$. Taking $m$ to be a protonic mass and considering a red shift factor $z \approx 10^{3}$ (so that $R_{0} \approx 10^{20} \mathrm{~cm}$ ), reasonable estimates of $a, b$ in (24) are $a \approx 10^{3}$ and $b \approx 10$. As illustrated by figures (1a), (1b) and (1c) below, we have positive and monotonously decreasing $T$ and $\dot{s}$, and monotonously increasing $\tau$, so that the evolution of a configuration of this type is consistent with the thermodynamical requirements (16)-(22). In fact, it is possible to show that conditions (22) hold for all trial functions of the form (24), provided $a, b$ comply with the constraint

$$
\frac{2}{3}\left(1+\frac{1}{b}\right)<1+\frac{1}{a}
$$

together with $k T_{0} / m c^{2}<1, Y \geq Y_{0}$, conditions satisfied by a large choice of empirical constants. For $n_{0}, T_{0}$ given by (24) and (25), we also have ${ }^{[14]}: \tau \propto 1 / n$ for $Y / Y_{0} \gg 1$, a form of $\tau$ qualitatively analogous to (18a) and to relaxation times of globular clusters $^{[17]}$. Further extension and development of the work presented in this paper is being undertaken. ${ }^{[14]}$. 


\section{Acknowledgments}

I acknowledge finantial support from CONACYT grant 3567E. I am grateful to H. Quevedo, J. Triginer and D. Pavón for useful suggestions and encouragement. Acknowledgement is also due to the memory of Deedee and Chocho, whose wise meowing contributed to clarify the author's ideas.

Figure Caption. The figures display: $\dot{s}$ (Figure (1a)), $T$ (Figure (1b)) and $\log _{10}(\tau)$ (Figure (1c)), ploted vs. $\arctan (r)$ and $\arctan (y)$ (for $y \geq 1$, where $y \equiv Y / Y_{0}$ ), for the spherical inhomogeneity described in the text. Notice that $\dot{s}$ and $\tau$ are everywhere positive and they respectively decrease/increase along increasing $y$ (roughly equivalent to the fluid worldlines). This example is not meant to be a model of a "real" gallaxy, but simply to illustrate that conditions (22) hold for physicaly reasonable initial value functions $n_{0}, T_{0}$. These plots were done with the symbolic package MapleV.

\section{References}

[1] Kramer D, Stephani H, MacCallum MAH., Herlt E 1980. Exact solutions of Einstein's field equations. Cambridge University Press, Cambridge, U.K. See also: Krasiński A 1997. Inhomogeneous cosmological models. Cambridge University Press, Cambridge, U.K.

[2] Dyer CC 1976, MNRAS, 175, 420. Raine DJ and Thomas EG 1981, MNRAS, 195, 649. Panek M 1992, Ap. J., 388, 225. Saez D, Arnau JV and Fullana MJ 1983, MNRAS, 263, 681.

[3] Jou D, Casas Vázqez J and Lebon G 1993. Extended Irreversible Thermodynamics.. Springer Verlag, Berlin, Heidelberg, New York.

[4] Hiscock W and Lindblom L 1983, Ann. Phys. (NY)., 151, 466.

[5] Israel W 1976, Ann. Phys. (NY)., 100, 30. See also: Israel W 1989, in Relativistic Fluid Dynamics. Eds. M. Anile and Y. Choquet-Bruhat, Spriger-Berlin.

[6] Jou D, Casas Vázqez J, Lebon G 1988, Rep. Prog. Phys., 51, 1104. See also: Pavón D, Jou D and Casas Vázqez J 1982, Ann. Inst. Henri Poincare A, 36, 79.

[7] Muller I and Ruggieri T 1993, Extended Thermodynamics., Springer Tracts in Natural Philosophy, Volume 37. Springer Verlag, Berlin, Heidelberg, New York.

[8] R. Maartens 1995, Class. Quantum Grav., 12, 1455.

[9] Hiscock WA and Salmonson J 1991, Phys. Rev. D, 43, 3249.

[10] Pavón D and Romano V 1993, Phys. Rev. D, 47, 1396. See also: Méndez V and Triginer J 1996, J. Math. Phys., 37, 2906.

[11] G. Wannier 1987. Statistical Physics.. Dover Publications Inc., New York.

[12] Zemansky MW 1957. Heat and Thermodynamics.. MaGraw-Hill Book Company, New York, Toronto, London. See chapter 8.

[13] Calvão MO and Lima JAS 1989, Phys. Lett. A, 141, 229. See also: Garecki J and Stelmach J 1990, Annals of Physics, 204, 315.

[14] Sussman RA and Triginer J 1998. In preparation.

[15] I. King 1962, The Astronomical Journal, 67.

[16] Galaxies, Quasars and Cosmology.. L.Z. Fang and R. Ruffini, Editors 1985. Advanced Series in Astrophysics and Cosmology, vol. 2. World Scientific Publishing Company, Pte. Ltd. See pp. 50-52.

[17] Relativity Theory and Astrophysics. 2. Galactic Structure.. J. Ehlers, Editor 1967. Lectures in Applied Mathematics, vol 9. American Mathematical Society. Providence, Rhode Island. See pp. 44-53. 


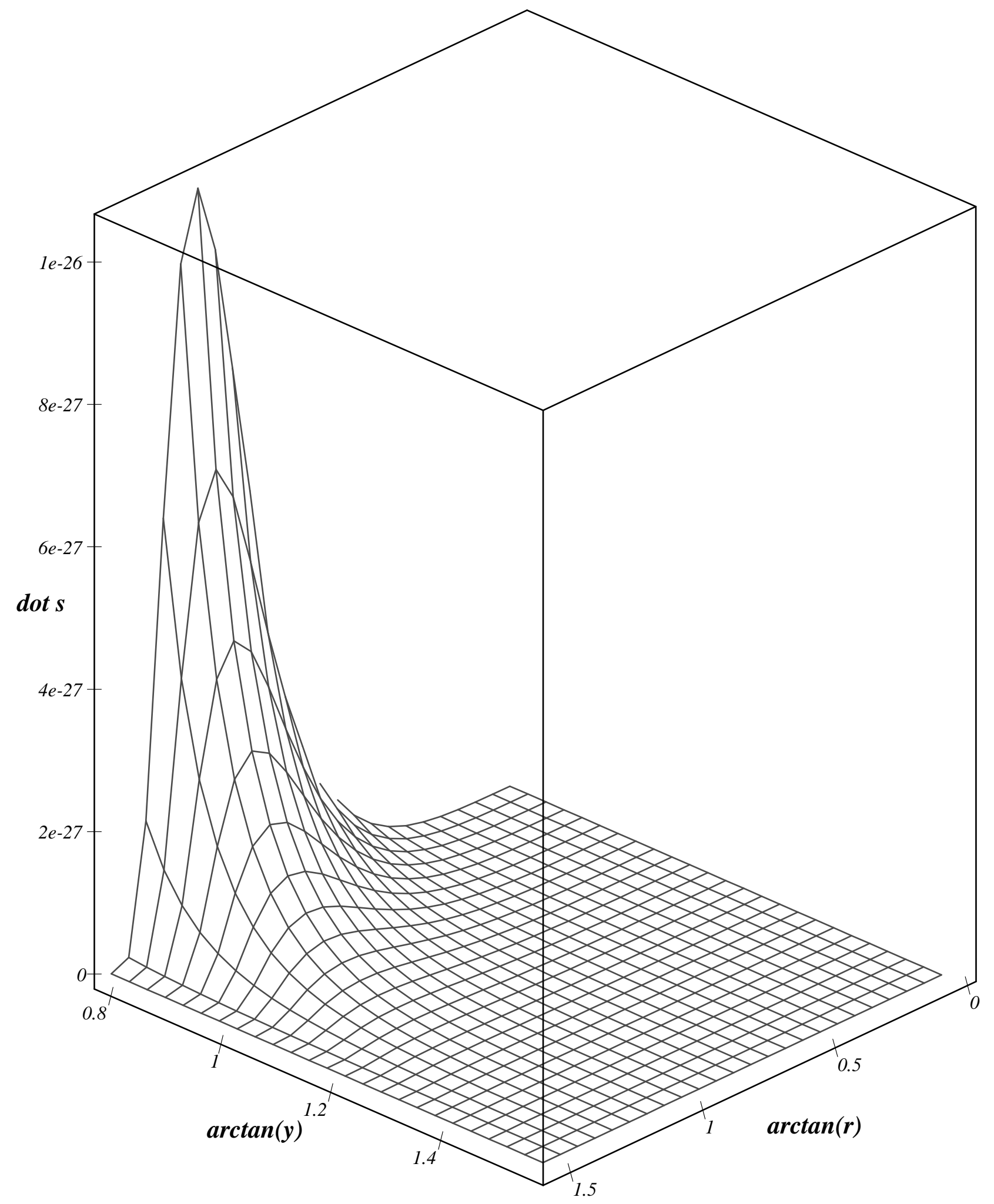




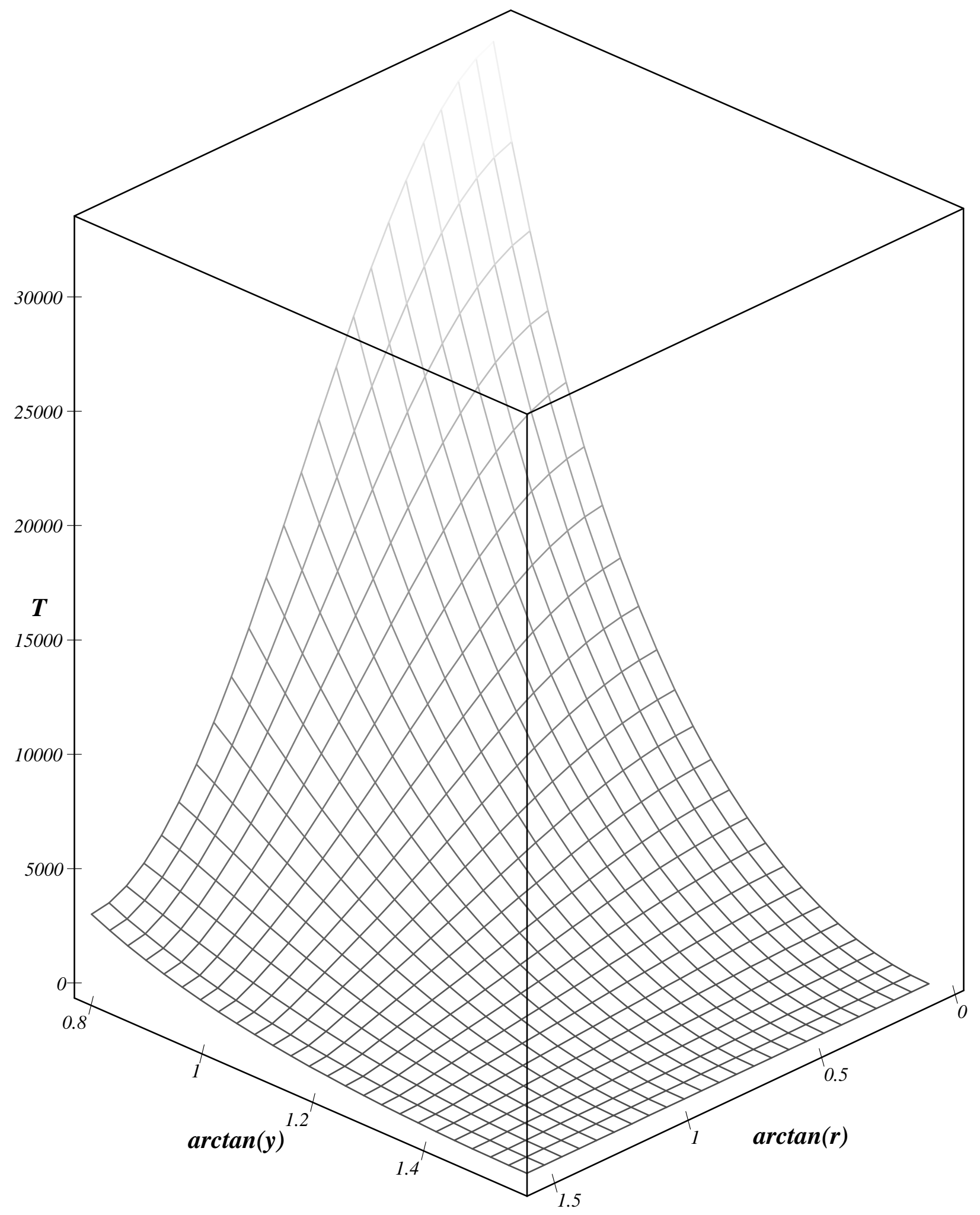


Figure (1c)

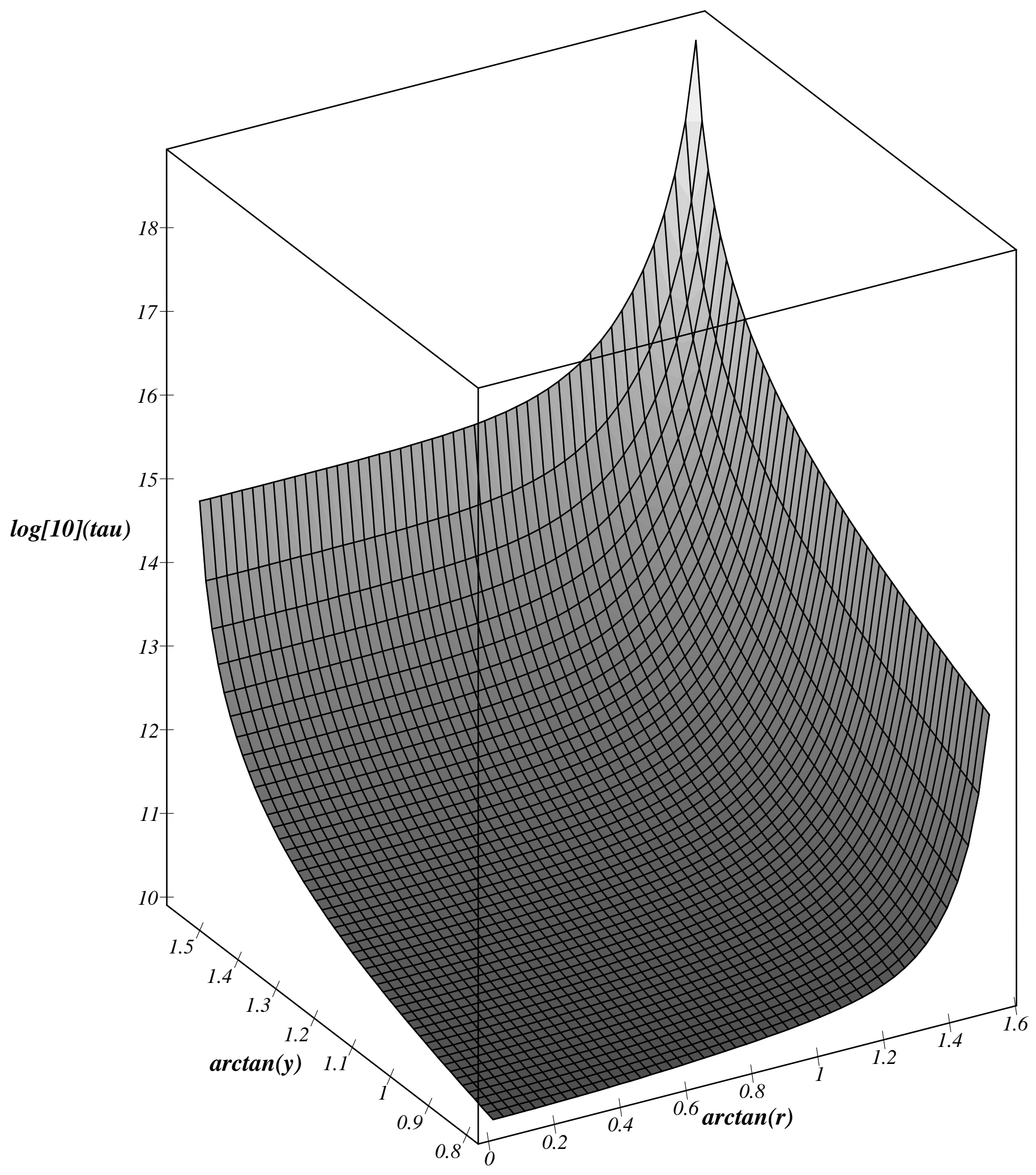

